\title{
Niveles séricos de 25-hidroxivitamina D y su relación con síndrome metabólico en una población de hombres jóvenes no diabéticos
}

\author{
Serum levels of 25-hydroxy vitamin D and their \\ relationship to metabolic syndrome in a population \\ of young nondiabetic men
}

\author{
Jhoan Sebastián Roncancio-Muñoz, María Camila Romero-Ortiz, \\ Sergio Andrés Vallejo-Ávila, Héctor Fabio Sandoval-Álzate, Yessica Agudelo-Zapata, \\ Juan Sebastián Suárez-Niño, luis Manuel Maldonado-Acosta, Roberto Franco-Vega, \\ María Fernanda Garcés-Gutiérrez, Antonio Iglesias-Gamarra, Jorge Eduardo Caminos-Pinzón \\ - Bogotá, D.C. (Colombia)
}

DOI: https://doi.org/10.36104/amc.2020.1323

\section{Resumen}

Introducción: los niveles no suficientes de vitamina D(VD) se han asociado a varias patologías no osteomusculares; sin embargo, es motivo de controversia si éstos se asocian a mayor prevalencia de síndrome metabólico (SM).

Objetivo: determinar y comparar la frecuencia de insuficiencia y deficiencia de 25-hidroxivitamina $\mathrm{D}(25(\mathrm{OH}) \mathrm{D})$ entre hombres jóvenes obesos no diabéticos y controles con peso normal, y su correlación con el estado de SM.

Material y métodos: estudio de corte transversal, que incluyó 62 individuos con peso normal y 47 en obesidad, se determinaron los niveles séricos de $25(\mathrm{OH}) \mathrm{D}$ y se midieron parámetros antropométricos y bioquímicos para establecer criterios de SM.

Resultados: de los 47 sujetos con obesidad, 25 tenían SM, mientas que ninguno de los sujetos de peso normal cumplía con dichos criterios. No se encontraron diferencias estadísticamente significativas en cuanto a la presencia de síndrome en correlación con los niveles de vitamina D ( $\mathrm{p}=0.94)$. La media de los niveles séricos de $25(\mathrm{OH}) \mathrm{D}$ para la población total fue $30.6 \pm 8.3 \mathrm{ng} /$ $\mathrm{mL}$; en sujetos normopeso $30.8 \pm 8.5 \mathrm{ng} / \mathrm{mL}$ y entre los obesos con $\mathrm{SM}$ fue $30.1 \pm 9.2 \mathrm{ng} / \mathrm{mL}$ y sin $\mathrm{SM}$ de $30.6 \pm 7.5 \mathrm{ng} / \mathrm{mL}$. Por otro lado no hubo una correlación significativa entre los parámetros individuales de síndrome metabólico y los niveles séricos de VD, tanto de manera global, como en el análisis por subgrupos.

Conclusión: no hubo una correlación significativa entre los niveles séricos de $25(\mathrm{OH}) \mathrm{D}$ con el estado de SM, tampoco se identificó ningún tipo de correlación significativa entre éstos y los parámetros antropométricos y bioquímicos estudiados. (Acta Med Colomb 2020; 45. DOI: https:// doi.org/10.36104/amc.2020.1323).

Palabras clave: vitamina $D$, síndrome metabólico, obesidad, obesidad metabólica benigna, resistencia a la insulina.

\section{Abstract}

Introduction: insufficient levels of vitamin D (VD) have been associated with several nonmusculoskeletal diseases. However, whether they are associated with a greater prevalence of metabolic syndrome (MS) is a matter of controversy.

Objective: to determine and compare the frequency of 25-hydroxy vitamin D $(25(\mathrm{OH}) \mathrm{D})$ insufficiency and deficiency in young, obese nondiabetic men and normal weight controls, and its correlation with metabolic syndrome.
Dr. Jhoan Sebastián Roncancio-Muñoz: Departamento de Medicina Interna; Dra. María Camila Romero-Ortiz: Servicio de Endocrinología, Departamento de Medicina Interna; Dr. Sergio Andrés Vallejo-Ávila: Departamento de Medicina Interna; Dr. Héctor Fabio SandovalÁlzate: Departamento de Nutrición Humana; Dra. Yessica Agudelo-Zapata: Servicio de Endocrinología, Departamento de Medicina Interna; Dr. Juan Sebastián Suárez-Niño: Departamento de Medicina Interna; Dr. Luis Manuel Maldonado-Acosta: Servicio de Endocrinología, Departamento de Medicina Interna; Dr. Roberto Franco-Vega: Servicio de Endocrinología, Departamento de Medicina Interna; Dra. María Fernanda Garcés-Gutiérrez: Departamento de Fisiología; Dr. Antonio Iglesias-Gamarra: Servicio de Reumatología, Departamento de Medicina Interna; Dr. Jorge Eduardo Caminos-Pinzón: Departamento de Fisiología. Universidad Nacional de Colombia. Bogotá, D.C. (Colombia).

Correspondencia: Dr. Sebastián Roncancio Muñoz. Jhoan Sebastian Roncancio Muñoz. Bogotá, D.C. (Colombia) E-mail: jsroncanciom@unal.edu.co Recibido: 04/VII/2019 Aceptado: 04/V/2020 
Material and methods: a cross-sectional study which included 62 normal weight and 47 obese individuals. Serum levels of $25(\mathrm{OH}) \mathrm{D}$ were ascertained and anthropometric and biochemical parameters were measured to establish MS criteria.

Results: of the 47 obese subjects, 25 had MS, while none of the normal weight subjects met the criteria. There were no statistically significant differences in the presence of the syndrome related to the vitamin D levels ( $\mathrm{p}=0.94$ ). The mean serum $25(\mathrm{OH}) \mathrm{D}$ level for the total population was $30.6 \pm 8.3 \mathrm{ng} / \mathrm{mL}$; in normal weight subjects it was $30.8 \pm 8.5 \mathrm{ng} / \mathrm{mL}$, in obese subjects with MS it was $30.1 \pm 9.2 \mathrm{ng} / \mathrm{mL}$, and in obese subjects without MS it was $30.6 \pm 7.5 \mathrm{ng} / \mathrm{mL}$. Furthermore, there was no significant correlation between the individual MS parameters and serum VD, either globally or on subgroup analysis.

Conclusion: there was no significant correlation between serum $25(\mathrm{OH}) \mathrm{D}$ levels and MS, nor was any significant correlation found between these and the anthropometric and biochemical parameters studied. (Acta Med Colomb 2020; 45. DOI: https://doi.org/10.36104/amc.2020.1323).

Key words: vitamin D, metabolic syndrome, obesity, metabolically benign obesity, insulin resistance.

\section{Introducción}

La vitamina D3 (VD) es una prohormona que se origina principalmente en la piel a partir del 7-dehidrocolesterol por cambios conformacionales asociados a irradiación por rayos ultravioleta $\mathrm{B}$, dependiendo de la intensidad y la duración de la exposición a éstos y varía en torno a la altitud y las estaciones climáticas (1-3). La VD puede también obtenerse a partir de la dieta o suplementos nutricionales, siendo estas fuentes de menor importancia biológica (4).

Posterior a la producción de VD, esta es transportada en el torrente sanguíneo por la proteína de unión a la vitamina D (DBP, por sus siglas en inglés; vitamin D binding protein), la cual es capaz de transportar todos los metabolitos asociados a la VD, teniendo mayor afinidad por la forma 25-hidroxivitamina D $(25(\mathrm{OH}) \mathrm{D})(2,5,6)$. El complejo $\mathrm{VD} / \mathrm{DBP}$ es transportado al hígado, donde ocurre la primera hidroxilación mediada fundamentalmente por la CYP2R1 produciéndose $25(\mathrm{OH}) \mathrm{D}$, a su vez, ésta es transportada al túbulo contorneado proximal del riñón donde se produce la segunda hidroxilación por parte de CYP27B1 sintetizándose así la 1.25-dihidroxivitamina $\mathrm{D}(1,25(\mathrm{OH}) 2 \mathrm{D} 3)$, forma biológicamente activa de la hormona $(1,2,7)$.

Las acciones biológicas de la 1.25(OH)2D3 clásicamente han estado ligadas al metabolismo mineral óseo; sin embargo, se han descrito nuevas funciones dentro de las que se incluyen la homeostasis del metabolismo energético, mediadas por la estimulación del receptor de VD, el cual modifica directamente la transcripción génica y la modulación directa de la función de complejos enzimáticos citoplasmáticos en diferentes tipos de células (1, 7-9).

No existe un consenso acerca de los niveles normales de $25(\mathrm{OH}) \mathrm{D}$; sin embargo, la mayoría de sociedades coinciden en definir una concentración sérica de $30 \mathrm{ng} / \mathrm{mL}$ o más como suficiente, $20-29 \mathrm{ng} / \mathrm{mL}$ insuficiente y menos de $20 \mathrm{ng} / \mathrm{mL}$ deficiente (10-12). Se estima que en el mundo cerca de un billón de personas tienen insuficiencia o deficiencia de VD. En Estados Unidos la prevalencia es 50-80\% de la población general, siendo más frecuente en los ancianos $(10,13,14)$. En Colombia se ha encontrado una prevalencia entre 55.1 y $16.6 \%$ de insuficiencia y deficiencia respectivamente en pacientes postmenopáusicas (15).

Las bajas concentraciones de VD se han relacionado de manera directa con raquitismo y osteoporosis, cáncer de colon, mama y próstata, enfermedades autoinmunes como esclerosis múltiples y diabetes mellitus tipo 1 , y enfermedades cardiovasculares como hipertensión arterial, diabetes mellitus tipo 2 y falla cardiaca $(13,16-19)$.

Por otro lado, el síndrome metabólico (SM) se ha definido como un grupo de alteraciones clínicas y de laboratorio, es considerado como un evento primario para el desarrollo de enfermedad ateroesclerótica $(20,21)$. La prevalencia global se encuentra entre 20 y $50 \%$, pero varía en torno a la población estudiada $(20,22,23)$. Los sujetos con SM tienen cinco veces más riesgo de diabetes mellitus tipo 2 , dos a cuatro veces más riesgo de infarto de miocardio y ataque cerebrovascular, dos veces más riesgo de enfermedad cardiovascular y mayor riesgo de mortalidad comparado con sujetos sin este (21, 24-27). Por lo tanto, la búsqueda de nuevos factores involucrados en la fisiopatología del SM, sobre todo aquellos modificables, ha aumentado en los últimos años.

Adicionalmente se ha encontrado una relación entre la concentración sérica de VD y la presencia de SM; sin embargo, la evidencia es controversial en este aspecto. Por lo tanto se planteó la realización de este estudio para caracterizar los niveles de V D y correlacionarlos con la presencia de SM y sus diferentes componentes; perímetro de cintura, glucosa, presión arterial, triglicéridos y colesterol de alta densidad (c-HDL por sus siglas en inglés), así como también con la concentración de proteína $\mathrm{C}$ reactiva (PCR).

\section{Material y métodos}

Este es un estudio de corte transversal, la muestra fue tomada por conveniencia e incluyo un total de 109 individuos. Todos los participantes fueron valorados por al menos 
un médico y un nutricionista para la evaluación clínica y toma de medidas antropométricas. De acuerdo con las guías actuales, la obesidad fue definida como un IMC mayor o igual a $30 \mathrm{Kg} / \mathrm{m}^{2}(28,29)$. Todos los sujetos con diagnóstico de diabetes mellitus, hipertensión arterial, enfermedad cardiovascular u otro tipo de enfermedades crónicas o que hubiesen consumido medicamentos de forma regular en el último año fueron excluidos del estudio.

Una muestra sanguínea fue tomada para medición de triglicéridos (TG), colesterol total (c-Total), c-HDL, colesterol de baja densidad (c-LDL por sus siglas en inglés), glucosa e insulina, el índice de resistencia a insulina HOMA-IR (Homeostasis Model Assessment - Insulin Resistance index) fue calculado por el método de Matthews y colaboradores (30), se determinó la concentración sérica de 25OHVD por técnica de electroquimioluminiscencia mediante un kit disponible comercialmente, de acuerdo con los protocolos del fabricante (LIAISON ${ }^{\circledR} 25 \mathrm{OH}$ Vitamin D Total Assay), la insuficiencia y deficiencia se definió como valores séricos entre $20-29 \mathrm{ng} / \mathrm{mL}$ y menores a $20 \mathrm{ng} / \mathrm{mL}$ respectivamente (10-11).

El diagnóstico de síndrome metabólico se estableció cuando se cumplían tres de los siguientes criterios; circunferencia de cintura mayor o igual a $90 \mathrm{~cm}$ (punto de corte recomendado para hombres sudamericanos), triglicéridos mayor o igual a $150 \mathrm{mg} / \mathrm{dL}$, colesterol HDL menor a $40 \mathrm{mg} / \mathrm{dL}$, presión arterial sistólica mayor o igual a $130 \mathrm{mmHg}$ o diastólica mayor o igual a $85 \mathrm{mmHg}$ y glucosa en ayunas mayor o igual a $100 \mathrm{mg} / \mathrm{Dl}(21,27)$.

El estudio fue aprobado por el comité de ética de la Facultad de Medicina de la Universidad Nacional de Colombia, todos los participantes recibieron la información completa acerca del protocolo del estudio, las preguntas fueron aclaradas y posteriormente se procedió a la firma del consentimiento informado.

Mediante pruebas de Shapiro-Wilk y Kolmogorov Smirnov se determinó normalidad para los grupos de comparación (síndrome metabólico o no SM). Posteriormente se obtuvieron medidas de tendencia central; media y desviación estándar para el grupo con distribución normal y mediana con rango intercuartil para el grupo no normal. Se realizó la prueba no paramétrica de Wilcoxon-Rank para comparar las variables entre los grupos en el caso de no normalidad y $t$-student en caso de normalidad, se tomó una $p<0.05$ para diferencias estadísticamente significativas.

Por último, se realizó la correlación de los dos grupos mediante Pearson en caso de variables con distribución normal y Spearman cuando no hay normalidad con su respectivo $p$-valor.

\section{Resultados}

Un total de 109 sujetos fueron reclutados en el estudio con una edad promedio de 23 años, de éstos, 62 tenían un peso en rango de normalidad (normopeso) y los 47 restantes en rango de obesidad. Dentro de los individuos obesos 25 cumplían con al menos tres criterios de SM (OSM+), mientras que los otros 22 cumplían con dos o menos (OSM-), en el grupo control de sujetos normopeso ninguno cumplía con criterios de SM.

En la Tabla 1 se describen las características basales de los tres grupos, el estudio antropométrico no encontró diferencia significativa en la talla; sin embargo, las medidas de peso, IMC, perímetro de cintura y cadera fueron significativamente menores entre el grupo de sujetos con peso normal comparado con ambos subgrupos de obesidad $(\mathrm{p}<0.01)$.

En cuanto a los parámetros para la definición de SM se encontró que el perímetro de cintura medio fue $109.8 \pm 7.8 \mathrm{~cm}$ en el grupo de OSM+ y de $105.9 \pm 18.1 \mathrm{~cm}$ en el grupo de OSM- no encontrándose una diferencia significativa entre estos $(\mathrm{p}=0.21)$; así mismo, ocurrió con los niveles de c-HDL $(42.8 \pm 1.9 \mathrm{mg} /$ $\mathrm{dL}$ vs $45.1 \pm 17.6 \mathrm{mg} / \mathrm{dL} \mathrm{p}=0.6$ ). Los parámetros que marcaron la diferencia entre obesos con SM y sin este fueron; la presión arterial sistólica $(133.6 \pm 9.7 \mathrm{mmHg}$ vs $121.2 \pm 14.4 \mathrm{mmHg}$ $\mathrm{p}=0.001)$, los niveles de glucosa plasmática $(93.6 \pm 12.5 \mathrm{mg} /$ $\mathrm{dL}$ vs $84 \pm 6.8 \mathrm{mg} / \mathrm{dL} \mathrm{p}=0.002$ ) y la presión arterial diastólica $(86.4 \pm 9.7 \mathrm{mmHg}$ vs $79.6 \pm 11.3 \mathrm{mmHg} \mathrm{p}=0.03)$.

Por otro lado, se encontró una elevación significativa en los valores de perímetro de cintura, presión arterial sistólica y diastólica, c-LDL y triglicéridos en los sujetos obesos sin criterios para SM cuando se comparó con sujetos de peso normal con un valor $\mathrm{p}<0.01$ para todas las comparaciones.

Al evaluar la concentración de insulina, se evidenció una diferencia significativa al comparar todos los grupos, encontrándose los valores más bajos en los individuos de peso normal, intermedios en OSM- y más altos en OSM+ siendo de $8.6 \pm 5.1 \mathrm{uUI} / \mathrm{mL}, 2.9 \pm 10.9 \mathrm{uUI} / \mathrm{mL}$ y $30.2 \pm 11.3$ uUI/Ml, respectivamente, ocurriendo este mismo fenómeno con el índice de resistencia a la insulina HOMA-IR $(1.8 \pm 1.2$, $4.8 \pm 2.5$ y $7 \pm 2.9$ respectivamente). Los niveles de PCR fueron significativamente más elevados en OSM+ respecto de los OSM- $(\mathrm{p}<0.05)$ y a su vez éstos fueron superiores a los sujetos con peso normal $(\mathrm{p}<0.01)$.

La media de los niveles séricos de 25-OHVD para la población total fue de $30.6 \pm 8.3 \mathrm{ng} / \mathrm{mL}$, se encontró que $46.8 \%$ de los sujetos se encontraba en rangos de suficiencia, el $45.9 \%$ en insuficiencia y $7.3 \%$ en deficiencia de esta hormona de acuerdo con la categorización previamente descrita.

La concentración plasmática media de VD fue de $30.8 \pm 8.5 \mathrm{ng} / \mathrm{mL}$ en sujetos normopeso, $30.6 \pm 7.5 \mathrm{ng} / \mathrm{mL}$ en OSM- y de $30.1 \pm 9.2 \mathrm{ng} / \mathrm{mL}$ en OSM+ no encontrándose una diferencia significativa entre los subgrupos estudiados $(\mathrm{p}=0.94)$ (Figura 1).

Por último, se realizó una matriz de correlación entre los componentes individuales del SM y la concentración sérica de 25-OHVD para cada grupo de obesos con resultados heterogéneos, los niveles más bajos de VD se relacionaron con mayor presión arterial sistólica, perímetro de cintura y concentraciones séricas de triglicéridos, sin embargo el 
Tabla 1. Comparación de las características antropométricas y metabólicas entre los subgrupos.

\begin{tabular}{|c|c|c|c|c|c|c|}
\hline Variables (unidades) & Normopeso & Obeso SM- & Obeso SM+ & $\mathbf{p}^{*}$ & $\mathbf{p}^{* *}$ & $\mathbf{p}^{* * *}$ \\
\hline Edad (años) & $23.1 \pm 3.40$ & $24.0 \pm 3.87$ & $24.5 \pm 5.19$ & 0.29 & 0.15 & 0.75 \\
\hline Talla (metros) & $1.73 \pm 0.05$ & $1.71 \pm 0.06$ & $1.75 \pm 0.06$ & 0.39 & 0.11 & 0.10 \\
\hline Peso (Kg) & $64.9 \pm 6.57$ & $105.9 \pm 18.14$ & $111.8 \pm 15.46$ & $<0.001$ & $<0.001$ & 0.24 \\
\hline $\operatorname{IMC}\left(\mathrm{Kg} / \mathrm{m}^{2}\right)$ & $21.6 \pm 1.49$ & $35.7 \pm 4.83$ & $36.5 \pm 4.69$ & $<0.001$ & $<0.001$ & 0.53 \\
\hline Perímetro de cintura $(\mathrm{cm})$ & $77.1 \pm 4.38$ & $106.7 \pm 8.78$ & $109.8 \pm 7.80$ & $<0.001$ & $<0.001$ & 0.21 \\
\hline Perímetro de cadera $(\mathrm{cm})$ & $93.4 \pm 4.44$ & $118.7 \pm 10.22$ & $119.9 \pm 9.92$ & $<0.001$ & $<0.001$ & 0.68 \\
\hline Relación cintura/cadera & $0.82 \pm 0.03$ & $0.90 \pm 0.03$ & $0.91 \pm 0.06$ & $<0.001$ & $<0.001$ & 0.23 \\
\hline PAS (mmHg) & $111.7 \pm 10.97$ & $121.2 \pm 14.45$ & $133.6 \pm 9.68$ & 0.001 & $<0.001$ & 0.001 \\
\hline PAD $(\mathrm{mmHg})$ & $70.3 \pm 8.33$ & $79.6 \pm 11.27$ & $86.4 \pm 9.67$ & $<0.001$ & $<0.001$ & 0.03 \\
\hline Glucosa basal (mg/dL) & $84.1 \pm 8.40$ & $84 \pm 6.81$ & $93.6 \pm 12.48$ & 0.95 & $<0.001$ & 0.002 \\
\hline $\mathrm{c}-\mathrm{HDL}(\mathrm{mg} / \mathrm{dL})$ & $47.1 \pm 6.81$ & $41.9 \pm 5.28$ & $42.8 \pm 11.94$ & 0.49 & 0.04 & 0.60 \\
\hline $\mathrm{c}-\mathrm{LDL}(\mathrm{mg} / \mathrm{dL})$ & $94.1 \pm 27.89$ & $115.3 \pm 25.59$ & $101.4 \pm 24.86$ & 0.002 & 0.25 & 0.07 \\
\hline $\mathrm{TG}(\mathrm{mg} / \mathrm{dL})$ & $94.8 \pm 31.56$ & $124.6 \pm 56.80$ & $205.7 \pm 57.5$ & 0.003 & $<0.001$ & 0.06 \\
\hline HOMA-IR & $1.80 \pm 1.22$ & $4.8 \pm 2.46$ & $7.04 \pm 2.90$ & $<0.05$ & $<0.01$ & $<0.01$ \\
\hline Insulina basal (uUI/mL) & $8.6 \pm 5.10$ & $22.9 \pm 10.95$ & $30.2 \pm 11.31$ & $<0.001$ & $<0.001$ & $<0.01$ \\
\hline PCR (mg/dL) & $1.36 \pm 3.21$ & $4.5 \pm 3 . .97$ & $4.5 \pm 3.76$ & $<0.01$ & $<0.01$ & $<0.05$ \\
\hline \multicolumn{7}{|c|}{$\begin{array}{l}\text { * Comparación entre sujetos con peso normal contra obesos sin síndrome metabólico. } \\
\text { ** Comparación entre sujetos con peso normal contra obesos con síndrome metabólico. } \\
\text { *** Comparación entre obesos sin síndrome metabólico contra obesos con síndrome metabólico. } \\
\text { Los valores son expresados en } n \pm D E ; \text { número } \pm \text { Desviación estándar. }\end{array}$} \\
\hline
\end{tabular}

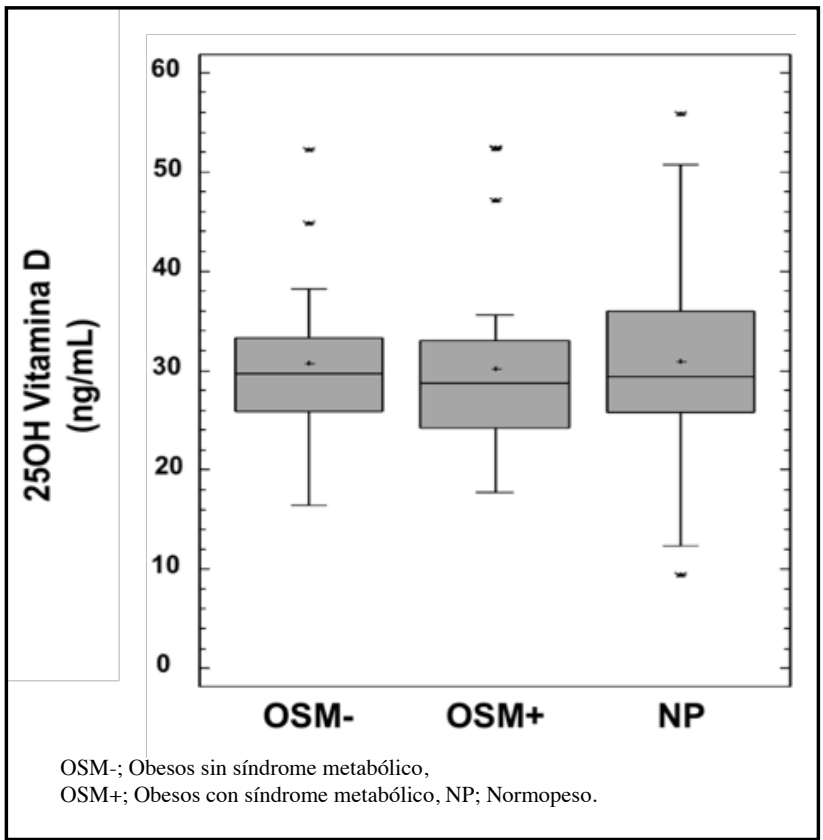

Figura 1. Box plot de la comparación entre subgrupos de la concentración sérica de vitamina $D$. grado de correlación fue débil, tanto en OSM- como en OSM+ (Figuras 2a, 2c y 2f), la glucosa sérica tuvo una correlación neutra con la concentración de VD en ambos grupos (Figura 2e), la presión arterial diastólica tuvo una correlación heterogénea siendo positiva en los OSM+ y negativa en OSM- (Figura $2 b$ ).

Finalmente, los niveles más altos de colesterol HDL se relacionaron con más altas concentraciones de vitamina $D$ en obesos sin SM, pero con menos concentraciones en obesos con SM (Figura 2d), a pesar de las tendencias, el grado de correlación fue débil para todas las variables.

\section{Discusión}

La prevalencia de SM dentro del grupo estudiado fue $21 \%$, siendo ligeramente inferior a la reportada por la Asociación Latinoamericana de Diabetes de 25-33\% (31), pero similar a la descrita en Colombia, en donde se informan datos muy variables que van desde 13.2 hasta $72.7 \%$, dependiendo de los criterios usados para definirlo (31-34), la obesidad central, la presión arterial sistólica elevada y los triglicéridos altos fueron los parámetros más frecuentemente afectados, acorde a lo descrito en otros trabajos (33). 

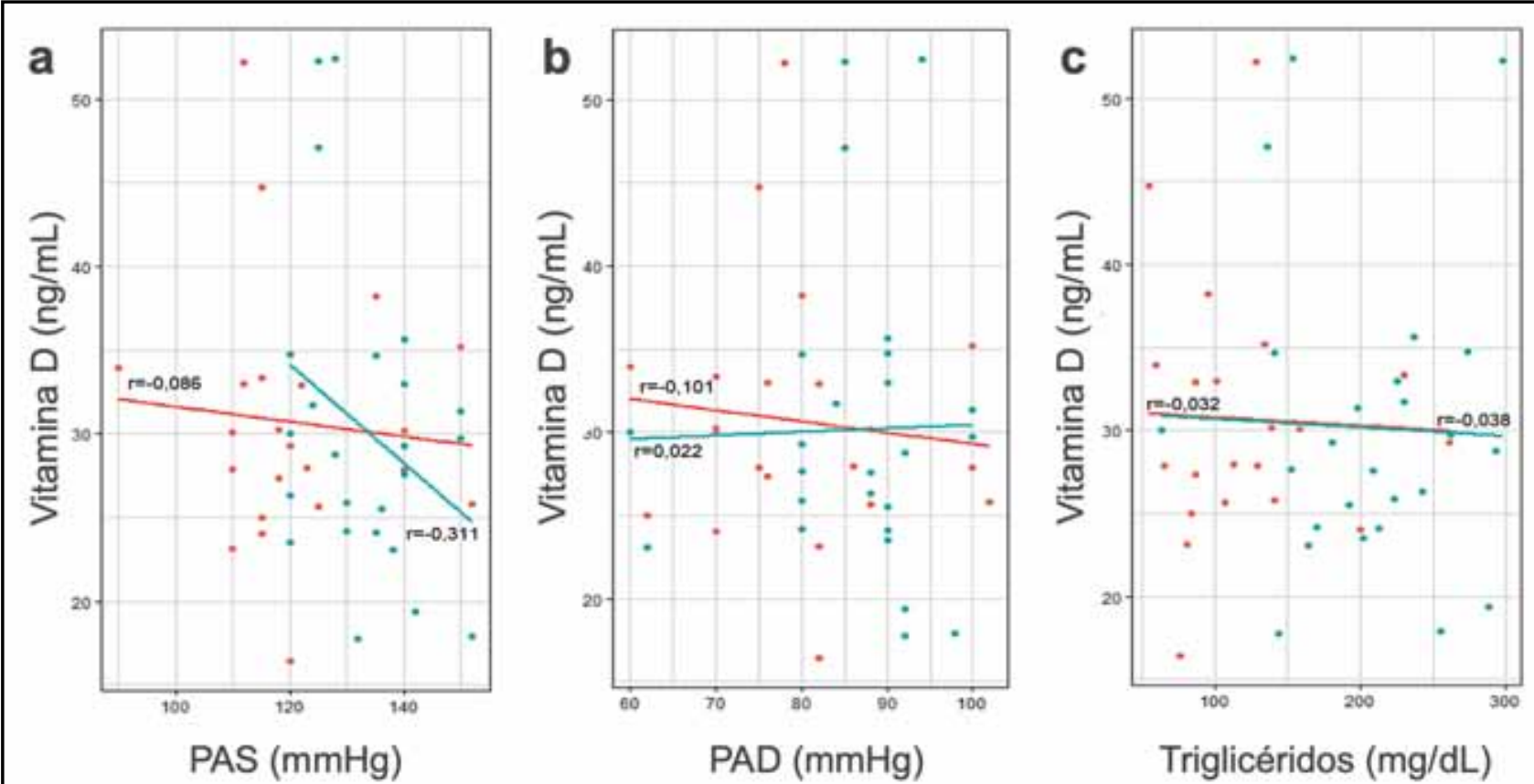

Triglicéridos (mg/dL)
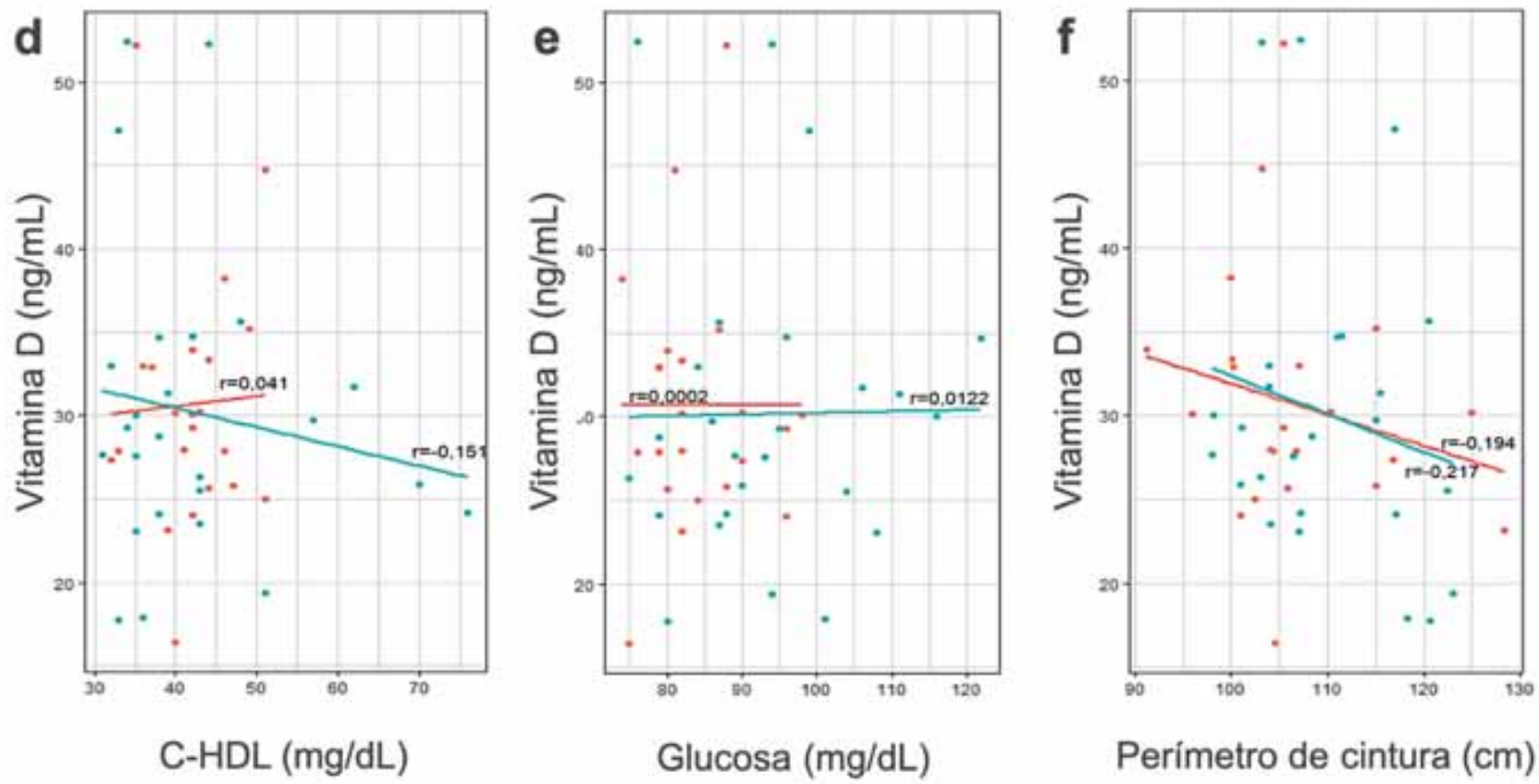

Perímetro de cintura $(\mathrm{cm})$

Figura 2. Diagrama de correlación entre la concentración de vitamina D y los componentes del síndrome metabólico en los pacientes obesos.

Diagrama de correlación entre los componentes individuales de síndrome metabólico (a) presión arterial sistólica, (b) presión arterial diastólica, (c) triglicéridos, (d) colesterol HDL (e) glucosa basal y $(f)$ perímetro de cintura y y la concentración sérica de vitamina $D$. La línea roja corresponde a los pacientes obesos sin síndrome metabólico y la línea azul a pacientes obesos con síndrome metabólico. Ver texto para más información.

Poca evidencia existe en Latinoamérica acerca del comportamiento de la concentración sérica de VD en adultos sanos; sin embargo, se considera que son mayores en los países del trópico dado un mayor tiempo de exposición solar $(35,36)$. Oliveri et al. reportan un déficit de 25-OHVD de 90\% en ancianos saludables en Argentina, con una menor prevalencia en el norte del país (37). Un estudio en mujeres postmenopáusicas con osteoporosis en México, Chile y Brasil encontró niveles inadecuados de VD en 67, 50 y $42 \%$ de las pacientes respectivamente $(35,38)$. En Colombia son escasos los datos reportados acerca de la prevalencia de hipovitaminosis, D. Molina y cols. evaluaron 205 mujeres 
postmenopáusicas con osteoporosis, encontrando que $71.7 \%$ cursaban con una concentración de VD menor a $30 \mathrm{ng} / \mathrm{mL}$, similar a lo descrito por Devía et al. con una prevalencia de $69.5 \%(39,40)$. Adicionalmente, Quintana et al. compararon pacientes con artritis reumatoide y controles sanos, encontrando una prevalencia de niveles inadecuados de 25-OHVD de 98.5 y $72.8 \%$ respectivamente (41). En nuestra población, $53.2 \%$ de los sujetos cursaban con concentraciones de VD menor a $30 \mathrm{ng} / \mathrm{Ml}$, siendo menor a lo descrito en Colombia, Argentina y México, esto explicado en parte por la menor edad de los pacientes evaluados, junto con una ausencia de comorbilidades, siendo este uno de los primeros estudios en evaluar el estado de la vitamina D sérica en población colombiana adulta joven sana.

Cuando se compararon los niveles séricos de 25-OHVD en los grupos de SM y no SM, no hay una diferencia significativa, no encontrándose de esta manera una relación entre el estado de insuficiencia y deficiencia de VD y la condición de SM. No hay consenso aún de una posible relación causal entre los niveles inadecuados de 25-OHVD y el riesgo de padecer SM y enfermedad cardiovascular $(42,43)$; sin embargo, algunos estudios predominantemente de corte transversal han encontrado una relación inversa entre estos niveles y el SM, así como sus componentes aislados, considerándose que dentro de los efectos extra óseos de la VD estaría la estimulación pancreática de la secreción de insulina, la inhibición del sistema renina angiotensina aldosterona y la estimulación de la secreción de óxido nítrico a nivel endotelial, entre otros, que explicarían un mayor riesgo de enfermedad cardiovascular entre estos pacientes $(1,43-46)$.

Por otro lado, acorde a los resultados del presente trabajo, varios estudios no han encontrado una relación significativa entre la concentración sérica de 25-OHVD y el estado metabólico así como con el riesgo de enfermedad cardiovascular, un metaanálisis publicado por Sang et al. no encontró una relación significativa en los estudios longitudinales, considerándose que la relación descrita en estudios transversales probablemente no es causal, y que influirían muchos factores de confusión siendo uno de los más importantes la obesidad (47).

Así mismo cuando se llevaron a cabo los estudios de correlación entre las variables independientes de SM y los niveles séricos de VD, no hubo una correlación con un poder suficiente para considerarla relevante, considerándose de esta manera que no hay dependencia entre estas variables, lo cual puede explicar parcialmente los resultados negativos en estudios de intervención acerca del uso de la terapia de suplementación con VD y el riesgo de desarrollar SM, hipertensión arterial, diabetes mellitus y enfermedad cardiovascular $(48,49)$.

Trabajos más recientes han mostrado una relación entre las bajas concentraciones de vitamina D y el riesgo de morbilidad y mortalidad de origen cardiovascular, evidenciándose hasta un aumento de $7 \%$ del riesgo de muerte cardiovascular con cada disminución de $4 \mathrm{ng} / \mathrm{mL}$ en la concentración plasmática de VD considerándose como un factor de riesgo cardiovascular no clásico susceptible de ser intervenido $(49,50)$; sin embargo, recientes ensayos clínicos y metaanálisis no han sido consistentes con mostrar un beneficio clínicamente significativo sobre los desenlaces fuertes en enfermedad cardiovascular $(51,52)$.

Dentro de las limitaciones del estudio, vale la pena recalcar la toma de muestra por conveniencia que no permite tener una representación fidedigna de la población general, así mismo la falta de datos acerca de la exposición solar. Por otro lado, las cambiantes definiciones de síndrome metabólico que han condicionado una epidemiología muy variable.

En conclusión, la baja concentración de 25-hidroxivitamina D no se asoció a mayor probabilidad de tener SM, ni tampoco hubo una correlación fuerte con las variables individuales de éste en la población estudiada, estos resultados aportan datos al cuerpo de evidencia existente a nivel global y en desarrollo a nivel nacional acerca de esta controversia, con lo cual apoya la independencia relativa del metabolismo energético y la falta de beneficios en la suplementación de 25-OHVD en prevención de enfermedad cardiovascular.

\section{Referencias}

1. Christakos S, Dhawan P, Verstuyf A, Verlinden L, Carmeliet G. Vitamin D: metabolism, molecular mechanism of action, and pleiotropic effects. Physiol Rev 2016; 96: 365-408.

2. Dusso AS, Brown AJ, Slatopolsky E. Vitamin D. Am J Physiol Renal Physiol 2005; 289: F8-F28

3. Webb AR, DeCosta BR, Holick MF. Sunlight regulates the cutaneous production of vitamin D3 by causing its photodegradation. J Clin Endocrinol Metab 1989; 68: 882-887.

4. Bikle DD. Vitamin D metabolism, mechanism of action, and clinical applications. Chemistry \& Biology 2014; 21: 319-329.

5. Cooke NE, Haddad JG. Vitamin D binding protein (Gc-globulin). Endocr Rev 1989; 10: 294-307.

6. Bouillon R, Van Assche FA, Van Baelen H, Heyns W, De Moor P. Influence of the vitamin D-binding protein on the serum concentration of 1,25-dihydroxyvitamin D3. Significance of the free 1,25-dihydroxyvitamin D3 concentration. J Clin Invest 1981; 67: 589-596

7. DeLuca HF. Overview of general physiologic features and functions of vitamin D. Am J Clin Nutr 2004; 80: 1689S-1696S.

8. Christakos S, Ajibade DV, Dhawan P, Fechner AJ, Mady, LJ. Vitamin D: Metabolism. Endocrinol Metab Clin N Am 2010; 30: 243-253.

9. Hii CS, Ferrante A. The non-genomic actions of vitamin D. Nutrients 2016; 8: $1-14$.

10. Holick MF, Binkley NC, Bischoff-Ferrari HA, Gordon CM, Hanley DA, Heaney RP, et al. Murad MH, Weaver CM. Evaluation, treatment, and prevention of vitamin D deficiency: An endocrine society clinical practice guideline. J Clin Endocrinol Metab 2011; 96: 1911-1930.

11. LeFevre ML. Screening for vitamin D deficiency in adults: U.S. preventive services task force recommendation statement. Ann Intern Med 2015; 162: 133-140.

12. Hanley DA, Cranney A, Jones G, Whiting SJ, Leslie WD, Cole DEC, et al. Vitamin D in adult health and disease: A review and guideline statement from osteoporosis Canada. CMAJ 2010; 182: E610-E618.

13. Holick MF. Vitamin D deficiency. N Engl J Med 2007; 357: 266-281.

14. Rosen CJ. Vitamin D insufficiency. N Engl J Med 2011; 364: 248-254.

15. Molina JF, Molina J, Escobar JA, Betancur JF, Giraldo A. Niveles de 25 hidroxivitamina D y su correlación clínica con diferentes variables metabólicas y cardiovasculares en una población de mujeres posmenopáusicas. Acta Med Colomb 2011; 36: 18-23.

16. Gorham ED, Garland CF, Garland FC, Grant WB, Mohr SB, Lipkin M, et al. Vitamin D and prevention of colorectal cancer. J Steroid Biochem Mol Biol 2005; 97: 179-194. 
17. Giovannucci E, Liu Y, Rimm EB, Hollis BW, Fuchs CS, Stampfer MJ, et al. Prospective study of predictors of vitamin D status and cancer incidence and mortality in men. J Natl Cancer Inst 2006; 98: 451-459.

18. Burgaz A, Orsini N, Larsson SC, Wolk A. Blood 25-hydroxyvitamin D concentration and hypertension: a meta-analysis. J Hypertens 2011; 29:636-645.

19. Motiwala SR, Wang TJ. Vitamin D and cardiovascular disease. Curr Opin Nephrol Hypertens 2011; 20: 345-353.

20. Kaur J. A comprehensive review on metabolic syndrome. Cardiol Res Pract 2014; 2014: 1-21

21. Samson SL, Garber AJ. Metabolic syndrome. Endocrinol Metab Clin N Am 2014; 43: 1-23

22.Pandit K, Goswami S, Ghosh S, Mukhopadhyay P, Chowdhury S. Metabolic syndrome in south asians. Indian J Endocrinol Metab 2012; 16: 44-55.

23. Beltrán-Sánchez H, Harhay MO, Harhay MM, McElligott S. Prevalence and trends of metabolic syndrome in the adult U.S. population, 1999-2010. J Am Coll Cardiol 2013; 62: 697-703.

24. Ford ES. The metabolic syndrome and mortality from cardiovascular disease and all-causes: Findings from the National Health and Nutrition Examination Survey II mortality study. Atherosclerosis 2004; 173: 309-314.

25. Mottillo S, Filion KB, Genest J, Joseph L, Pilote L, Poirier P, et al. The metabolic syndrome and cardiovascular risk: A systematic review and meta-analysis. J Am Coll Cardiol 2010; 56: 1113-1132.

26. Wilson PW, D'Agostino RB, Parise H, Sullivan L, Meigs JB. Metabolic syndrome as a precursor of cardiovascular disease and type 2 diabetes mellitus. Circulation 2005; 112: 3066-3072.

27. Alberti KG, Eckel RH, Grundy SM, Zimmet PZ, Cleeman JI, Donato KA, et al. Harmonizing the metabolic syndrome: a joint interim statement of the International Diabetes Federation Task Force on Epidemiology and Prevention; National Heart, Lung, and Blood Institute; American Heart Association; World Heart Federation; International Atherosclerosis Society; and International Association for the Study of Obesity. Circulation 2009; 120: 1640-1645.

28. Jensen MD, Ryan DH, Apovian CM, Ard JD, Comuzzie AG, Donato KA, et al.2013 AHA/ACC/TOS guideline for the management of overweight and obesity in adults: a report of the American College of Cardiology/American Heart Association Task Force on Practice Guidelines and The Obesity Society. J Am Coll Cardiol 2014; 63: 2985-3023.

29. Garvey WT, Garber AJ, Mechanick JI, Bray GA, Dagogo-Jack S, Einhorn D, et al. American association of clinical endocrinologists and american college of endocrinology position statement on the 2014 advanced framework for a new diagnosis of obesity as a chronic disease. Endocr Pract 2014; 20: 977-989.

30. Matthews DR, Hosker JP, Rudenski AS, Naylor BA, Treacher DF, Turner RC. Homeostasis model assessment: Insulin resistance and $\beta$-cell function from fasting plasma glucose and insulin concentrations in man. Diabetologia 1985; 28 : 412-419.

31. Rosas J, González A, Aschner P, Bastarrachea R, et al. Epidemiología, Diagnóstico, Control, Prevención y Tratamiento del Síndrome Metabólico en Adultos. Consensos ALAD 2010; 18: 25-44

32. Ramirez-Velez R, González-Ruiz K, Correa-Bautista JE. Adiposidad corporal y su relación con componentes del síndrome metabólico en adultos de Bogotá, Colombia. Nutr Hosp 2015; 32: 1468-1475.

33. Dávila EP, Quintero MA, Orrego ML, Ford ES, Walke H, Arenas MM, et al. Prevalence and risk factors for metabolic syndrome in Medellin and surrounding municipalities, Colombia, 2008-2010. Prev Med 2012; 56: 30-34.

34. González-Zapata LI, Deossa GC, Monsalve-Álvarez J, Díaz-García J, Babio
N, Salas-Salvadó J. Metabolic syndrome in healthcare personnel of the university of antioquia-colombia; LATINMETS study. Nutr Hosp 2013; 28: 522-531.

35. Mithal A, Wahl DA, Bonjour JP, Burckhardt P, Dawson-Hughes B, Eisman JA, et al. Global vitamin D status and determinants of hypovitaminosis D. Osteoporos Int 2009; 20: 1807-1820.

36. Lips P. Worldwide status of vitamin D nutrition. J Steroid Biochem Mol Biol 2010; 121: 297-300.

37. Oliveri B, Plantalech L, Bagur A, Wittich AC, Rovai G, Pusiol E, et al. High prevalence of vitamin D insufficiency in healthy elderly people living at home in Argentina. Eur J Clin Nutr 2004; 58: 337-342.

38. Lips P, Hosking D, Lippuner K, Norquist JM, Wehren L, Maalouf G, et al. The prevalence of vitamin D inadequacy amongst women with osteoporosis: An international epidemiological investigation. J Intern Med 2006; 260: 245-254.

39. González DD, Zúñiga LC, Kattah CW. Insuficiencia de vitamina D en pacientes adultos con baja masa ósea y osteoporosis en la fundación Santa fe de Bogotá 2008-2009. Rev Colomb Reumatol 2010; 17: 212-218.

40. Quintana-Duque M, Caminos J, Varela-Nariño A, Calvo-Paramo E, Yunis J, Iglesias-Gamarra A. The role of 25-hydroxyvitamin D as a predictor of clinical and radiological outcomes in early onset rheumatoid arthritis. J Clin Rheumatol 2016; 23: 33-39.

41. Parker J, Hashmi O, Dutton D, Mavrodaris A, Stranges S, Kandala NB, et al. Levels of vitamin D and cardiometabolic disorders: systematic review and meta-analysis. Maturitas 2010; 65: 225-236.

42. Vitezova A, Zillikens MC, van Herpt TT, Sijbrands EJ, Hofman A, Uitterlinden AG, et al. Vitamin D status and metabolic syndrome in the elderly: The Rotterdam Study. Eur J Endocrinol 2015; 172: 327-335.

43. Bea JW, Jurutka PW, Hibler EA, Lance P, Martínez ME, Roe DJ, et al. Concentrations of the vitamin D metabolite $1,25(\mathrm{OH}) 2 \mathrm{D}$ and odds of metabolic syndrome and its components. Metabolism 2015; 64: 447-459.

44. Prasad P, Kochhar A. Interplay of vitamin D and metabolic syndrome: A review. Diabetes Metab Syndr 2016; 10: 105-112.

45. Ju SY, Jeong HS, Kim DH. Blood vitamin D status and metabolic syndrome in the general adult population: A dose-response meta-analysis. J Clin Endocrinol Metab 2014; 99: 1053-1063.

46. Pilz S, Verheyen N, Grübler MR, Tomaschitz A, März W. Vitamin D and cardiovascular disease prevention. Nat Rev Cardiol 2016; 13: 404-417.

47. Elamin MB, Abu Elnour NO, Elamin KB, Fatourechi MM, Alkatib AA, Almandoz JP, et al. Vitamin D and cardiovascular outcomes: A systematic review and meta-analysis. J Clin Endocrinol Metab 2011; 96: 1931-1942.

48. Bikle DD. Vitamin D metabolism, mechanism of action, and clinical applications. Chemistry \& Biology 2014 Mar 20; 21(3): 319-329.

49. Webb AR, DeCosta BR, Holick MF. Sunlight regulates the cutaneous production of vitamin D3 by causing its photodegradation. The Journal of clinical endocrinology and metabolism. 1989 May; 68(5): 882.

50. Wang T, Liu Z, Fu J, Min Z. Meta-analysis of vitamin D supplementation in the treatment of chronic heart failure. Scandinavian Cardiovascular Journal. 2019 May; 53(3): 110-116.

51. Scragg R, Stewart AW, Waayer D, Lawes CM, Toop L, Sluyter, et al. Effect of Monthly High-Dose Vitamin D Supplementation on Cardiovascular Disease in the Vitamin D Assessment Study. A Randomized Clinical Trial. JAMA Cardiol 2017 Apr; 2(6):608-616.

52. Yang J, Ou-Yang J, Huang J. Low serum vitamin D levels increase the mortality of cardiovascular disease in older adults: A dose-response meta-analysis of prospective studies. Medicine (Baltimore) 2019 Aug; 98(34): e16733. 